\title{
Simultaneous temperature and strain measurements performed by a step-changed arc-induced long-period fiber grating
}

\author{
Gaspar Rego, Rosane Falate, Oleg Ivanov, and José Luís Santos
}

\begin{abstract}
A compact sensor based on step-changed arc-induced long-period fiber gratings was implemented to discriminate between temperature and strain. The proposed sensor consists of a single long-period grating with two sections written consecutively in the SMF-28 fiber using the electric arc discharge technique. The two sections have the same period but different fabrication parameters. The operation of the sensor relies on the existence of a difference between the values of temperature and strain sensitivity of two neighboring resonances observed in the spectrum of the step-changed grating. The temperature and strain resolutions obtained for the sensor are $0.2{ }^{\circ} \mathrm{C}$ and $35 \mu \varepsilon$, respectively. (C) 2007 Optical Society of America

OCIS codes: $\quad 060.2370,050.2770,060.2340$.
\end{abstract}

\section{Introduction}

Long-period fiber gratings (LPFGs), in particular, arc-induced LPFGs can be used as sensing elements for simultaneous measurements of temperature and strain. ${ }^{1-4}$ Usually, to create a sensor based on an LPFG able to discriminate between two or more parameters, a grating whose spectrum has several resonances exhibiting different sensitivities to those parameters is employed. This principle was used by Bhatia et $a l .{ }^{1}$ for strain and temperature discrimination. However, the sensing schemes proposed previously have some drawbacks: the requirement of having two optical sources or two different fiber types, or the use of polarized light and/or polarization maintaining fibers. Recently, it was demonstrated that the response of arc-induced gratings to

G. Rego is with the Escola Superior de Tecnologia e Gestão, Avenida do Atlântico, 4900-348 Viana do Castelo, Portugal and with O. Ivanov (olegivvit@yandex.ru) and J. L. Santos at the Unidade de Optoelectrónica e Sistemas Electrónicos, Instituto de Engenharia de Sistemas e Computadores do Porto, Rua do Campo Alegre 687, 4169-007 Porto, Portugal. J. L. Santos is also with the Departamento de Física, Faculdade de Ciências da Universidade do Porto, Rua do Campo Alegre 687, 4169-007 Porto, Portugal. R. Falate is with the Sector Ciências Exatas e Naturais, Departamento de Informática, Avenida General Carlos Cavalcanti, 47481, Uvaranas 84030-900-Ponta Grossa, Brazil.

Received 8 September 2006; accepted 2 November 2006; posted 10 November 2006 (Doc. ID 74863); published 1 March 2007.

0003-6935/07/091392-05\$15.00/0

C 2007 Optical Society of America strain and temperature could be controlled by changing the gratings' fabrication parameters. ${ }^{5}$ By employing the properties of those gratings, a sensor head consisting of two concatenated LPFGs with cladding mode stripping in between was created for temperature and strain measurements. An index matching gel was applied to the fiber to strip cladding modes in order to eliminate recoupling in the second grating. Unfortunately, the use of gel strongly limits the range of working temperature. A possibility to extend the working range is to recoat the fiber in the region between the two gratings, but this is a poor alternative. Another disadvantageous feature of such a sensing head is its large overall length, which is undesirable for most applications.

In this paper, we study a more compact sensor, which does not have the drawbacks of the previous schemes. The sensor consists of an arc-induced LPFG with two sections written consecutively without any separation between them. The two sections are written in the same SMF-28 fiber with the same period but with different fabrication parameters.

\section{Gratings Fabrication and Characterization}

LPFGs for the sensor were written in Corning SMF-28 fiber using the electric arc technique. ${ }^{6}$ Our previous study ${ }^{5,7}$ has shown that the resonance wavelength positions of arc-induced LPFGs depend strongly on the fabrication parameters such as fiber pulling tension $T$, electric current $I$, arc duration $t$, and grating period $\Lambda$. In particular, it was observed that the increase in the electric current or in the arc 
duration shifted the spectra toward lower wavelengths, while higher fiber pulling tension results in an opposite shift of the spectra. In addition, an increase in the electric current, the arc duration, or the pulling tension makes the gratings' resonances deeper.

We found that the strain and temperature sensitivities also depend on the fabrication parameters. When the electric current changes from 9 to $12 \mathrm{~mA}$, the temperature sensitivity varies between 66 and $73 \mathrm{pm} /{ }^{\circ} \mathrm{C}$ [Fig. 1(a)], and, at the same time, the strain sensitivity of the LPFG's resonances (which appear in the wavelength range between 1.52 and $1.57 \mu \mathrm{m}$ ) varies considerably between -0.05 and $-0.36 \mathrm{pm} / \mu \varepsilon$ [Fig. 1(b)]. It should be emphasized that such properties of LPFGs produced by the electric arc technique have not to our knowledge been demonstrated so far by any other fabrication technique. In fact, as will be discussed below, the dependence of the resonance wavelengths on temperature is slightly nonlinear and the values averaged over the range between $20{ }^{\circ} \mathrm{C}$ and $110{ }^{\circ} \mathrm{C}$ are given in the figure. We also noticed that the nonlinearity is larger for larger values of the electric current, and the changes in the pulling tension affect the temperature sensitivity.
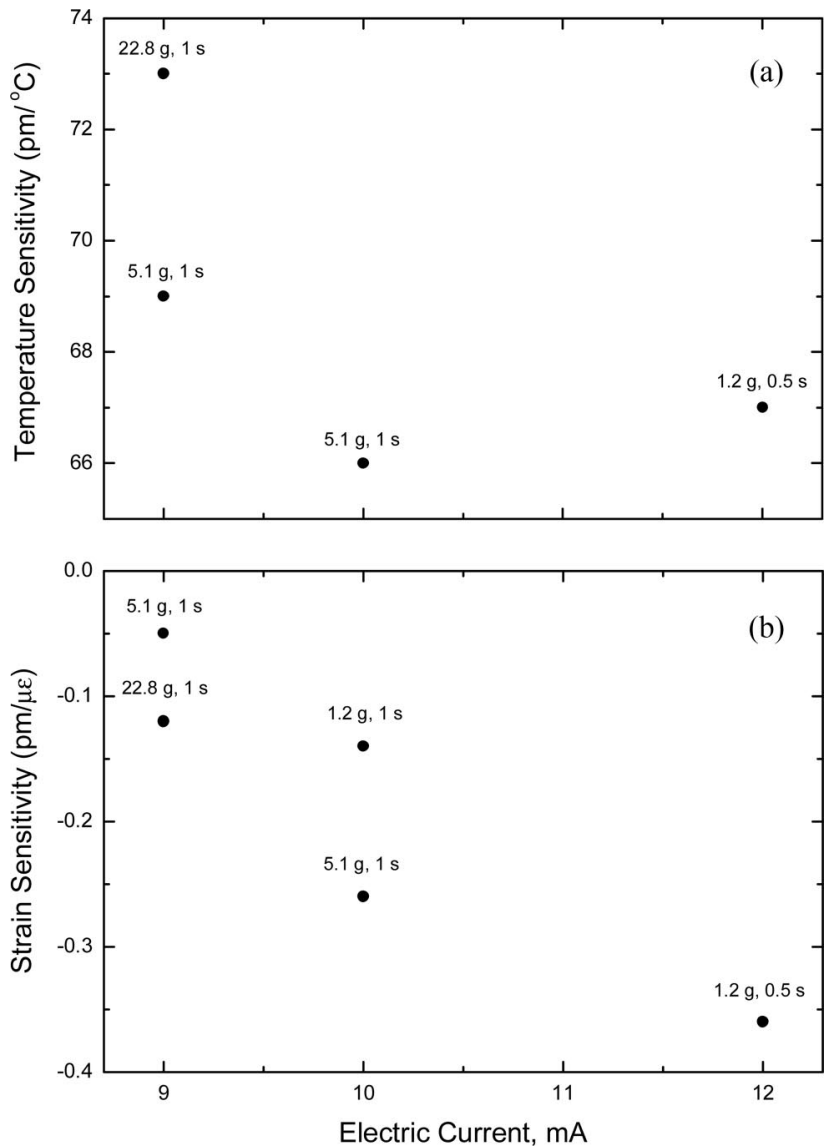

Fig. 1. (a) Temperature and (b) strain sensitivities of the fourth resonance of $540 \mu \mathrm{m}$ LPFGs as a function of electric current (corresponding pulling tension and arc duration are given near each point).
We cannot fully explain how and why temperature and strain sensitivities depend on the fabrication parameters; at the same time we may suggest some mechanisms that may cause such dependence. The inscription of arc-induced gratings reduces periodically the fiber cross section. This geometric change leads to a decrease in the effective refractive indices of the fundamental mode and, to a smaller extent, of the low-order cladding modes. As a result, the waveguide dispersion and, hence, the strain and temperature sensitivities are changed. ${ }^{8}$ For example, as follows from our estimation, a diameter reduction of $10 \%$ can lead to a change in the strain sensitivity as large as 35\%. Even larger changes can be attributable to annealing of intrinsic elastic and viscoelastic stresses caused by the arc discharge, which leads to considerable changes in the effective refractive indices via the photoelastic effect..$^{9,10}$ The magnitude of these changes can be comparable to the corecladding refractive index difference, and, therefore, larger changes in the strain sensitivity may be induced.

The temperature and strain sensitivities are also determined by the grating period, the order of the
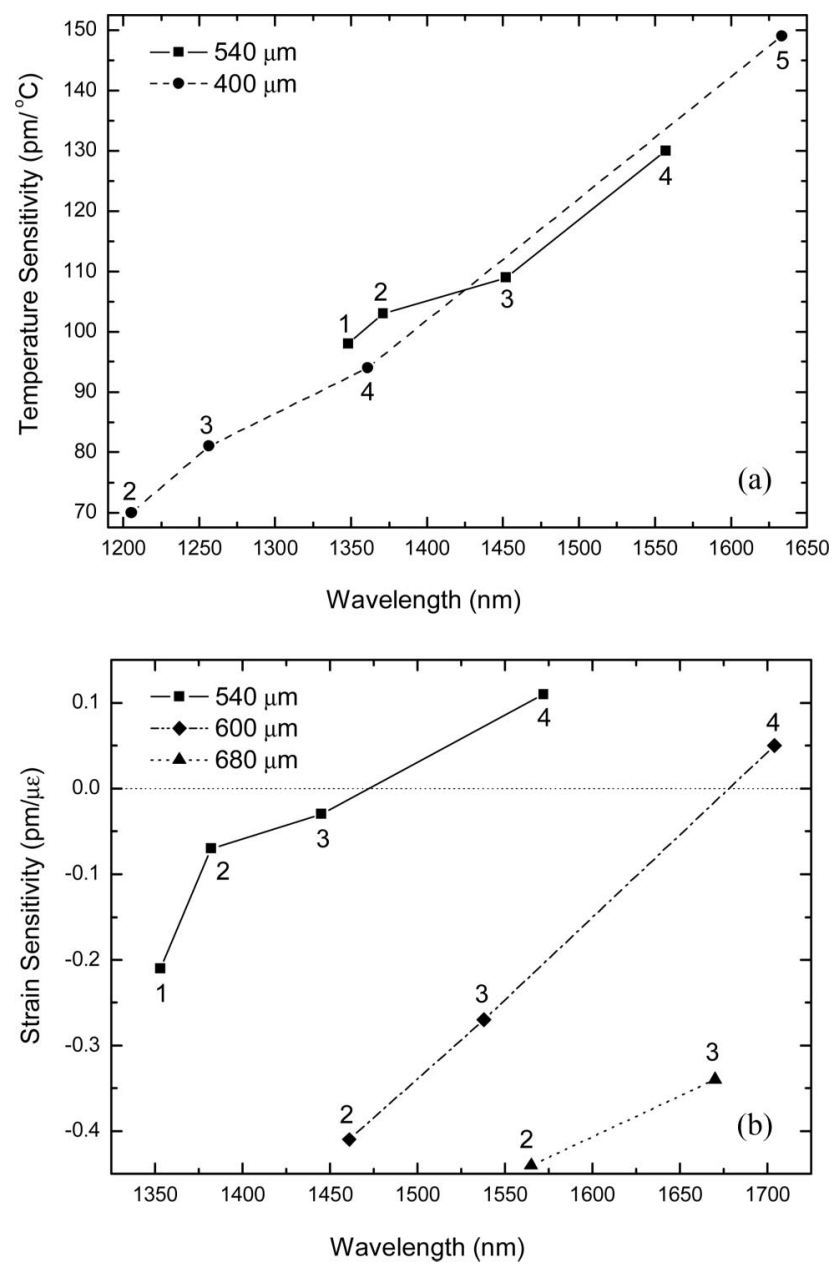

Fig. 2. (a) Temperature and (b) strain sensitivities versus resonance wavelengths for gratings with several different periods. The numbers indicate orders of resonances in the spectrum. 
cladding mode, and the resonance wavelength. It is well known that for a particular period the temperature sensitivity of LPFGs increases with the order of the cladding mode. ${ }^{5,7,11}$ For a particular resonance, the temperature sensitivity increases with the grating period, mostly because of an increase in the resonance wavelength. This is demonstrated in Fig. 2(a) where the temperature sensitivities of several cladding mode resonances with increasing mode number are given versus the resonance wavelength for two gratings having different periods. The dependence of temperature sensitivity of the cladding modes shown in the figure is almost linear. Regarding the strain sensitivity, its algebraic value increases with the order of the cladding mode (which agrees with Ref. 12) and decreases for a particular resonance with an increase in the grating period, as shown in Fig. 2(b) (similarly to UV-induced gratings). ${ }^{13}$

It follows from the results presented above, that different sensitivities to strain and temperature can be obtained for the same resonance produced by gratings fabricated with different parameters. By combining two such gratings and measuring the wavelength positions of two cladding mode resonances, the discrimination between strain and temperature can be achieved.

\section{Sensor Fabrication and Characterization}

To implement the sensor, an LPFG with two concatenated sections was induced by arc discharges in Corning SMF-28 fiber. The fabrication parameters were chosen such that the resonance wavelengths of the two sections were both approximately $1.55 \mu \mathrm{m}$ and exhibited different strain and temperature sensitivities. At the first stage a section containing 15 periods was inscribed with the following parameters: $T=22.8 \mathrm{~g}, I=9 \mathrm{~mA}, t=1 \mathrm{~s}$, and $\Lambda=540 \mu \mathrm{m}$. Then the pulling tension and the electric current were changed: $T=1.2 \mathrm{~g}$ and $I=11 \mathrm{~mA}$, and the second section containing 40 periods was written without any physical separation from the first section. The number of periods in the second section was larger because this grating should have strength similar to the strength of the first grating, which was produced with significantly lower tension.

Figure 3(a) shows two transmission spectra of the grating: one after writing the first section (dashed curve, S1) and the other after writing both sections (solid curve, $\mathrm{S} 1+\mathrm{S} 2$ ). The first spectrum, which belongs to the first section alone, has one broad resonance centered at $\sim 1580 \mathrm{~nm}$. During the writing process of the second section, the amplitude and bandwidth of this resonance increase with the dip position shifting to shorter wavelengths, and then the resonance splits in two. The writing process was stopped when the amplitude of the resonance at shorter wavelengths became almost equal to the amplitude of the resonance at longer wavelengths. The figure also shows the final spectrum produced by the stepchanged grating containing both sections. This spectrum has two major dips designated as Dip1 (at $1548 \mathrm{~nm}$ ) and Dip2 (at $1578 \mathrm{~nm}$ ). The position of the
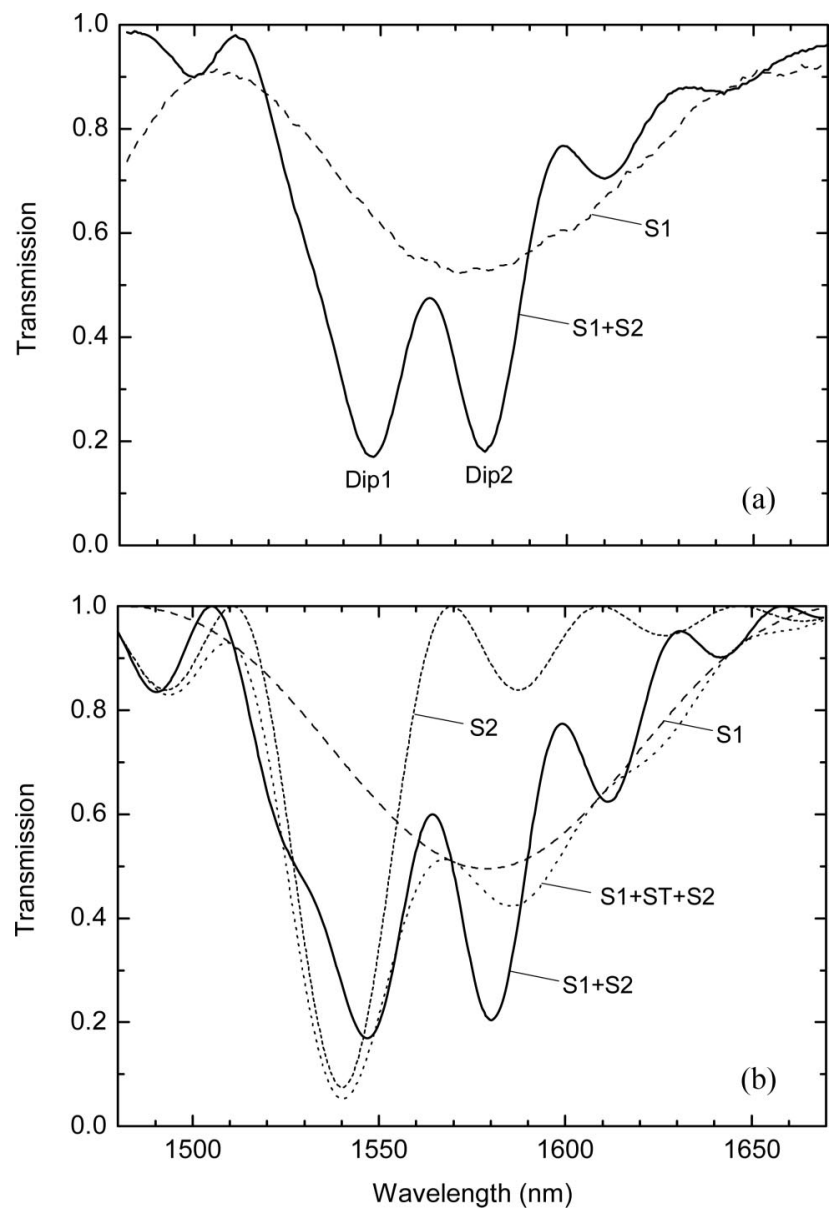

Fig. 3. (a) Measured spectra of the grating during the fabrication process: after writing the first section (dashed curve, S1) and after writing the second section (solid curve, S1 + S2). (b) Theoretical spectra of each of the two sections simulated separately (dashed and short dashed curves, S1 and S2), combined grating (solid curve, $\mathrm{S} 1+\mathrm{S} 2$ ), and cascaded grating with cladding mode stripped between the two sections (dotted curve, $\mathrm{S} 1+\mathrm{ST}+\mathrm{S} 2$ ).

dip at the longer wavelength is close to the resonance of the first section and is caused mostly by this section, while the resonance at the shorter wavelength is attributable mostly to the second section.

Although the shape of the resulting spectrum resembles the spectrum of a $\pi$-shifted grating, it is quite different since the spectrum is formed by two gratings written under different conditions. The sensitivities of two resonances of a $\pi$-shifted LPFG to various physical parameters are identical, which makes them inapplicable for discrimination between several measurands. At the same time, the stepchanged grating is different from the fiber structure consisting of two concatenated gratings between which the cladding mode is stripped. ${ }^{5}$ There is no interference effect in the latter cascaded grating, which results in a transmission spectrum that is a simple product of the two spectra of separate gratings.

Our step-changed grating is in fact very similar to the step-changed grating used for gain flattening ${ }^{14}$ and also to UV-induced gratings postexposed on 
half a region. ${ }^{15}$ The transmission through the stepchanged grating can be found from the transmission coefficients of the two constituent sections:

$$
T=\left|t_{\mathrm{co}}^{(1)} t_{\mathrm{co}}^{(2)} \exp (i \psi)+t_{\mathrm{cl}}^{(1)} t_{\mathrm{cl}}^{(2)}\right|^{2},
$$

where $t_{\mathrm{co}, \mathrm{cl}}^{(1,2)}$ are the amplitude transmission coefficients to the core and cladding modes for the first and second gratings. Since different fabrication parameters were used for the inscription of the two sections, a phase shift may also be induced between the two sections, and it is described in Eq. (1) by the parameter $\psi$. Knowing the spectrum of the first section, we used this expression to fit the resulting spectrum of the grating by adjusting the position and amplitude of the second section. The result of this simulation is shown in Fig. 3(b). The dashed (S1) and short dashed (S2) curves show the spectra of the first and second sections of the grating simulated separately. The dip position in the spectrum of the second section is at $1540 \mathrm{~nm}$, and the dip itself is twice lower than the dip of the first section. The solid curve $(\mathrm{S} 1+\mathrm{S} 2)$ is the simulated spectrum of the step-changed grating. It is seen that the theoretical curve correctly reproduces the two main dips and some other features of the experimental transmission spectrum. For comparison, the dotted curve $(\mathrm{S} 1+\mathrm{ST}+\mathrm{S} 2)$ demonstrates what would be the spectrum of the grating if the cladding mode were stripped between the two sections. The difference of this spectrum from the spectrum with interference consists in the unequal amplitudes of the two main dips and the smaller amplitude of modulation with wavelength.

When temperature and strain are applied to the step-changed grating the resonance dips of the two separate sections shift, the shift of the first section being different from the shift of the second one. As a result, the spectrum of the combined structure is modified. In particular, there are shifts in the resonance wavelengths of the two major dips. These shifts can be measured and used for simultaneous strain and temperature sensing.

To calibrate the sensor, we measured its response to the applied strain in the range 800-2400 $\mu \varepsilon$ and temperature in the range $22^{\circ} \mathrm{C}-110{ }^{\circ} \mathrm{C}$. The strain increased in steps of $200 \mu \varepsilon$ at a constant temperature of $22^{\circ} \mathrm{C}$, and temperature was changed in steps of $10{ }^{\circ} \mathrm{C}$ keeping a constant strain of $55 \mu \varepsilon$. At each step, the transmission spectrum of the grating was recorded using an optical spectrum analyzer. The results of calibration are presented in Fig. 4. Figure 4(a) shows the strain dependence of the wavelengths of the two main dips. The dependence for the dip at the shorter wavelengths is linear, while the dip at a longer wavelength appears to be insensitive to strain. This behavior is seemingly a result of the combined effect of the shifts of the two grating sections. The dependence of the resonance wavelengths on temperature, which is shown in Fig. 4(b) is slightly parabolic with greater slope for Dip2 and higher nonlinearity
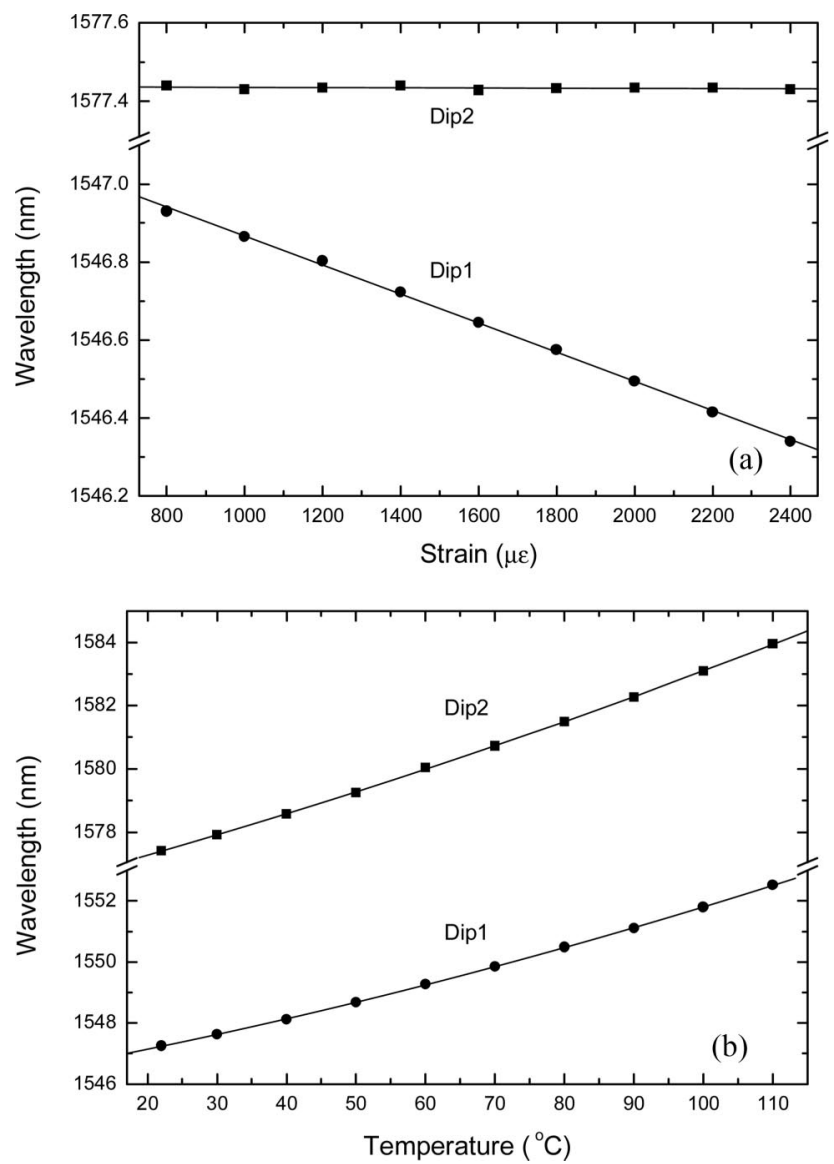

Fig. 4. (a) Strain and (b) temperature responses of the sensor. Experimental data points are fitted by linear (for strain) and quadratic (for temperature) functions.

for Dip1. Such a nonlinear dependence has also been previously observed. . $7,11^{-1}$

Since the dependences of the two dip wavelengths on strain are close to linear functions, and the dependences on temperature are close to quadratic functions, we represent these dependences as

$$
\left(\begin{array}{l}
\lambda_{1} \\
\lambda_{2}
\end{array}\right)=\left(\begin{array}{l}
\lambda_{10} \\
\lambda_{20}
\end{array}\right)+\left[\begin{array}{ll}
a_{1} & b_{1} \\
a_{2} & b_{2}
\end{array}\right]\left(\begin{array}{c}
\varepsilon \\
\Delta T
\end{array}\right)+\left[\begin{array}{ll}
0 & c_{1} \\
0 & c_{2}
\end{array}\right]\left(\begin{array}{c}
\varepsilon^{2} \\
\Delta T^{2}
\end{array}\right)
$$

where $\lambda_{10}$ and $\lambda_{20}$ are the dip wavelengths at the initial point $(\varepsilon=0, \Delta T=0), \Delta T=T-T_{0}$, and $T_{0}=$ $22{ }^{\circ} \mathrm{C}$. By optimizing the values of $\lambda_{i 0}, a_{i}, b_{i}$, and $c_{i}$, it is possible to fit dependence (2) to the experimental data. The best fit is for

$$
\begin{aligned}
\left(\begin{array}{l}
\lambda_{10} \\
\lambda_{20}
\end{array}\right) & =\left(\begin{array}{l}
1547.251 \\
1577.414
\end{array}\right)\left[\begin{array}{ll}
a_{1} & b_{1} \\
a_{2} & b_{2}
\end{array}\right]=10^{-3}\left[\begin{array}{cc}
-0.377273 & 47.9454 \\
0.010555 & 62.8258
\end{array}\right], \\
\left(\begin{array}{l}
c_{1} \\
c_{2}
\end{array}\right) & =10^{-6}\left(\begin{array}{l}
1.37254 \\
1.29529
\end{array}\right),
\end{aligned}
$$

when the wavelength is in nanometers, the temperature is in ${ }^{\circ} \mathrm{C}$, and the strain is in $\mu \varepsilon$.

To perform simultaneous measurement of two parameters, we should obtain the values of strain and 


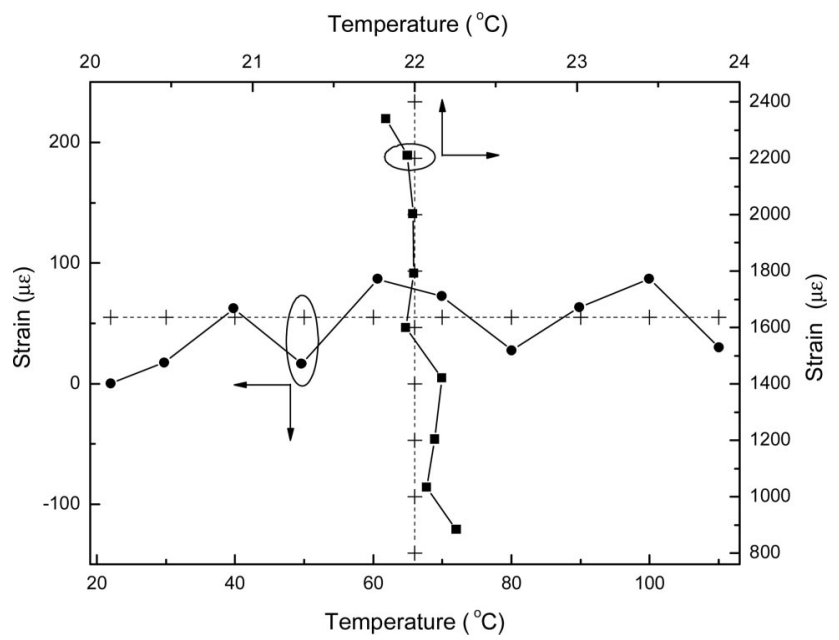

Fig. 5. Sensor output for the calibration points.

temperature from measured wavelengths. This can be realized by solving system (2) with respect to the unknown variables of strain and temperature:

$$
\begin{aligned}
\Delta T & =\frac{-b_{[1} a_{2]}+\sqrt{\left(b_{[1} a_{2]}\right)^{2}+4 c_{[1} a_{2]} \Delta \lambda_{[1} a_{2]}}}{2 c_{[1} a_{2]}}, \\
\varepsilon & =\frac{c_{[1} \Delta \lambda_{2]}-c_{[1} b_{2]} \Delta T}{c_{[1} a_{2]}},
\end{aligned}
$$

where $x_{[1} y_{2]}=x_{1} y_{2}-x_{2} y_{1}$, and $\Delta \lambda_{i}=\lambda_{i}-\lambda_{i 0}$. System (2) contains terms quadratic in strain and, therefore, has two solutions, the second solution being nonphysical.

The sensor resolution was estimated using the data obtained for the calibration. Figure 5 shows the result of the discrimination between strain and temperature for the calibration points. Each point in this figure was calculated from the experimental data of $\Delta \lambda_{1}$ and $\Delta \lambda_{2}$ using Eq. (4). The horizontal dependence is for the case of constant strain of $55 \mu \varepsilon$ and increasing temperature. The deviation of points from the crosses of the horizontal dashed line $(55 \mu \varepsilon)$ indicates the strain and temperature errors of the sensor. The vertical dependence is for the case of constant temperature of $22{ }^{\circ} \mathrm{C}$ and increasing strain. The rms deviations of temperature and strain from experimental values are found to be $0.2{ }^{\circ} \mathrm{C}$ and $35 \mu \varepsilon$, respectively.

Since the proposed sensor is limited at high temperatures only by the onset of stress relaxation, we assume that it can operate at temperatures as high as $700{ }^{\circ} \mathrm{C}$, and even higher temperatures may be reached if the fiber is annealed after the grating inscription.

\section{Conclusion}

A step-changed arc-induced LPFG consisting of two concatenated sections having the same period but different fabrication parameters was written in SMF-28 fiber. The transmission spectrum of the combined grating consisted of two major dips, which exhibited different temperature and strain sensitivities. Based on such a difference, a sensor for simultaneous measurement of those physical quantities was implemented. The proposed sensor demonstrated resolutions of $0.2{ }^{\circ} \mathrm{C}$ in temperature and $35 \mu \varepsilon$ in strain.

\section{References}

1. V. Bhatia, D. Campbell, R. O. Claus, and A. M. Vengsarkar, "Simultaneous strain and temperature measurement with long-period gratings," Opt. Lett. 22, 648-650 (1997).

2. O. Frazão, G. Rego, F. M. Araújo, L. A. Ferreira, H. M. Salgado, and J. L. Santos, "Simultaneous measurement of strain and temperature based on polarization loss properties of arcinduced long period gratings," in Second European Workshop on Fiber Sensors, Proc. SPIE 5502, 168-171 (2004).

3. K. J. Han, Y. W. Lee, J. Kwon, S. Roh, J. Jung, and B. Lee, "Simultaneous measurement of strain and temperature incorporating a long-period fiber grating inscribed on a polarization-maintaining fiber," IEEE Photon. Technol. Lett. 16, 2114-2116 (2004).

4. Y.-G. Han, S. Lee, C.-S. Kim, J. Kang, U.-C. Paek, and Y. Chung, "Simultaneous measurement of temperature and strain using dual long-period fiber gratings with controlled temperature and strain sensitivities," Opt. Exp. 11, 476-481 (2003).

5. G. Rego, P. S. Marques, H. M. Salgado, and J. L. Santos, "Simultaneous measurement of temperature and strain based on arc-induced long-period fibre gratings," Electron. Lett. 41, $60-62(2005)$.

6. G. Rego, O. Okhotnikov, E. Dianov, and V. Sulimov, "High temperature stability of long-period fiber gratings produced using an electric arc," J. Lightwave Technol. 19, 1574-1579 (2001).

7. G. Rego, P. S. Marques, H. M. Salgado, and J. L. Santos, "Arc-induced long-period fiber gratings," Fiber Integr. Opt. 24, 245-259 (2005).

8. X. Shu, L. Zhang, and I. Bennion, "Sensitivity characteristics of long-period fiber gratings," J. Lightwave Technol. 20, 255266 (2002)

9. F. Dürr, G. Rego, P. V. S. Marques, S. L. Semjonov, E. M. Dianov, H. G. Limberger, and R. P. Salathé, "Tomographic stress profiling of arc-induced long-period fiber gratings," J. Lightwave Technol. 23, 3947-3953 (2005).

10. G. Rego, F. Dürr, P. V. S. Marques, and H. G. Limberger, "Strong asymmetric stresses arc-induced in preannealed nitrogen-doped fibres," Electron. Lett. 42, 334-335 (2006).

11. G. Humbert and A. Malki, "Electric-arc-induced gratings in nonhydrogenated fibers: fabrication and high-temperature characterizations," J. Opt. A 4, 194-198 (2002).

12. O. Ivanov, "Coupling of hybrid modes in strained and heated fibers," Opt. Commun. 239, 311-321 (2004).

13. V. Bhatia, "Applications of long-period gratings to single and multiparameter sensing," Opt. Express 4, 457-466 (1999).

14. B. O. Guan, A. P. Zhang, H. Y. Tam, H. L. W. Chan, C. L. Choy, X. M. Tao, and M. S. Demokan, "Step-changed long-period fiber gratings," IEEE Photon. Technol. Lett. 14, 657-659 (2002).

15. Y.-G. Han and S. B. Lee, "Effect of initial coupling strength on transmission characteristics of long-period fiber grating and its sensing application," in Optical Fiber Sensors, Proc. SPIE 5855, 675-678 (2005). 